\title{
Endometrial beta3 Integrin profile reflects endometrial receptivity defects in women with unexplained recurrent pregnancy loss
}

\author{
Ariane Germeyer ${ }^{1 *}$, Ricardo F Savaris ${ }^{2}$, Julia Jauckus ${ }^{1}$ and Bruce Lessey ${ }^{3}$
}

\begin{abstract}
Background: The pathophysiology of recurrent pregnancy loss (RPL) is still unknown in 50\% of the cases. Herein we measure the expression of beta3 integrin subunit, a well-known implantation marker, in women with or without RPL and correlate it with the histological dating of the endometrial tissue.

Methods: LH-timed endometrial biopsies were obtained from cases (RPL; $n=21$, age 33.9+/-4.7) and healthy controls ( $n=29$; age 29.8+/-4.1) during the mid-secretory phase (post ovulatory day: 8 to 10). Endometrial samples were timed histologically according to Noyes' criteria and underwent immunohistochemical staining for beta3 integrin expression. For statistical analysis the semi-quantitative HSCORE was assessed. Type I (beta3 negative in an out-of-phase endometrium) and Type II defect (beta3 negative in an in-phase endometrium) were also analysed. Statistical analysis was done with Student $t$-test, Mann Whitney $U$ test, ANCOVA and chi square for trend. Significance was set as $P<0.05$.
\end{abstract}

Results: The mean (SD) age in controls was lower compared to cases [(29.8 (4.1) vs. 33.9 (4.7) - $P=0.001$; Student $t$-test)]. The median (range) expression of beta3 integrin in controls and cases was 1.94 (0 to 3.5) vs. 0.82 (0 to 3.6), respectively ( $P=0.001$; Mann Whitney $U$ test). Significance was still significant after adjusting for age $(P=0.03 ; A N C O V A)$. The normal positive staining $>=0.7$ of beta3 integrin subunit and in-phase endometrium was seen in 24 out of 29 $(82.8 \%)$ controls, but in only 6 out of 21 (28.6\%) of cases with RPL; Type I and II defects were seen in 10.3 and $6.9 \%$ of controls, while present in 52.4 and $19.1 \%$ of cases, respectively ( $P=0.0005$; chi-square).

Conclusions: Women with unexplained RPL had significantly reduced integrin expression compared to controls. Our findings underline the need for further molecular analysis of endometrial tissue in affected women.

Keywords: Beta3 integrin, Recurrent pregnancy loss, Endometrial dating

\section{Background}

Recurrent pregnancy loss (RPL), determined as two or more consecutive abortions by some authors, are seen in $1-3 \%$ of couples; an underlying cause, however, is only found in up to $50 \%$ [1]. These include embryonic factors, like poor quality embryos with or without karyotype abnormalities, as well as maternal factors, such as uterine malformations, general maternal infections, as well as local inflammation [2], hormonal abnormalities, immunogenic abnormalities (like thyroid antibodies, cardiolipin

\footnotetext{
* Correspondence: ariane.germeyer@med.uni-heidelberg.de

'Department of Gynecological Endocrinology and Reproductive Medicine, University Hospital Heidelberg, Heidelberg, Germany

Full list of author information is available at the end of the article
}

antibodies or antinuclear antibodies), genetic imbalances and thrombophilic diseases [3].

However, when none of these factors are evident, the recurrent pregnancy losses are classified as idiopathic, because the underlying mechanisms are not well understood. The functional expression of endometrial genes and proteins have been examined, because they compromise the endometrial microenviroment and may therefore contribute to an abnormal foetal-maternal interaction, resulting in pregnancy failure [4]. Furthermore synchronisation between embryonic development and endometrial decidualisation is found to be essential for adequate implantation [5]. Many authors have studied the embryo-endometrium interface in order to 
elucidate the pregnancy failure [6,7]. Global gene expression analysis $[8,9]$ has determined the adequate endometrial gene and protein expression during the short endometrial receptive stage in the mid-secretory phase, the so-called window of implantation (WOI) [10]. One of these proteins is the $\alpha v \beta 3$ integrin [11]. The combined integrin $\alpha v \beta 3$ acts as an adhesion promoter via cell-cell interactions and it has been very well characterized within the human endometrium [12]. The $\alpha v \beta 3$ integrin is expressed in the glandular epithelium during the window of implantation and translocates into endometrial stroma, if pregnancy occurs [13]. Reduced expression of $\alpha v \beta 3$ has been related to unexplained infertility [11] and in women with endometriosis [14]. $\mathrm{Xu}$ et al., in a recent publication, did not find any difference in $\beta 3$ integrin expression in women with RPL compared to normal fertile women [15]. However, these authors analysed the expression of $\beta 3$ integrin in formalin fixed paraffin embedded (FFPE) samples. It is known that $\beta 3$ integrin expression in FFPE produces artefacts and should be avoided [16]. In order to find a more accurate expression of $\beta 3$ integrin subunit in cases with RPL, would be necessary to use frozen sections of endometrial biopsies, instead of FFPE. Other authors reported the expression of av $\beta 3$ integrin in endometrialfrozen section and did not find any difference between groups $[17,18]$. Contrary, other authors found a reduction of $\alpha v \beta 3$ integrin during the implantation window in patients with recurrent pregnancy loss, compared to controls, either in frozen sections [19], or in microarray studies [7]. Possible discrepancies among studies could be related to technical differences. Our group has been using the SSA6 antibody to identify the $\beta 3$ integrin subunit, and the expertise developed in our group to evaluate $\beta 3$ immunostaining has been validated using $\mathrm{NIH}$ software image analysis [20]. This study has two objectives: a) to examine the expression of the $\alpha v \beta 3$ integrin in women with RPL compared to healthy controls, b) to compare integrin expression with morphologic dating of the endometrium, which has been widely used to judge the endometrial development and receptivity.

\section{Methods}

\section{Samples collection and processing}

This retrospective case-control study enrolled 21 women with identified unexplained RPL and 29 healthy controls. Demographics are depicted in Table 1. Inclusion criteria of "cases" were 2 or more pregnancy losses in the presence of normal thyroid function, anticardiolipin antibodies, lupus anticoagulant and uterine anatomy. Women with known uterine factors such as fibroids, uterine septa or intrauterine synechia were excluded. Other exclusion criteria were hormonal imbalance (anovulation, polycystic ovary syndrome, diabetes or untreated thyroid disease) and known immunologic, chromosomal or thrombophilic abnormalities. Controls were obtained from regularly cycling, normal fertility proven volunteers, without use of oral contraceptive in the previous 3 months. Endometrial biopsies were obtained from mid-secretory phase, 8-10 days after a urinary LH surge. These biopsies were obtained in sterile conditions, using a pipelle suction curettage at the outpatient gynaecology clinic in Heidelberg, Germany and in Chapel Hill, NC, USA. Endometrial biopsies were divided into 2 portions: one was formalin fixed and paraffin embedded for histological examination with haematoxylin and eosin analysis (for endometrial dating) and the other was frozen in liquid nitrogen for immunohistochemistry assessment of $\beta 3$ subunit expression. This study was submitted and approved by the Institutional Review Boards of both institutions (Greenville University Medical Center \& University of Heidelberg).

\section{Immunohistochemical staining}

Immunostaining for $\beta 3$ integrin subunit was performed on endometrial biopsies on $7 \mu \mathrm{m}$ cryosections using the

Table 1 Characteristics of the population

\begin{tabular}{|c|c|c|c|}
\hline Characteristics & Control $n=29$ & Recurrent pregnancy loss $n=21$ & $P$ value \\
\hline Age (y) mean (SD) & $29.8(4.1)$ & $33.9(4.7)$ & $0.001^{a}$ \\
\hline N. of miscarriage mean (SEM) & & $3.5(0.3)$ & \\
\hline \multicolumn{4}{|l|}{ Endometrial dating } \\
\hline in/out-of-phase (n) & $26 / 3$ & $10 / 11$ & $0.001^{b}$ \\
\hline \multicolumn{4}{|l|}{ Defect type $^{c}$ n (\%) } \\
\hline In phase with $\beta 3+$ & $24(82.8)$ & $6(28.6)$ & $0.0005^{b}$ \\
\hline Type I & $3(10.3)$ & $11(52.4)$ & \\
\hline Type ॥ & $2(6.9)$ & $4(19.1)$ & \\
\hline
\end{tabular}

anpaired Student $t$ test.

${ }^{\mathrm{b}} \mathrm{Chi}$-square for trend.

${ }^{c}$ In phase endometrium has positive $\beta 3$ integrin subunit.

Type I defect is an out-of-phase endometrium and $\beta 3$ integrin subunit expression is negative.

Type II defect is an "in-phase" endometrium and $\beta 3$ integrin subunit expression is negative. 
Vectastain Elite ABC kits (Vector Laboratories, Burlingame, CA) simultaneously in cases and controls in a same batch. Diaminobenzidine (Sigma, St. Louis, MO) was used as chromogen. All procedures were done simultaneously Tissue sections were fixed in $4 \%$ paraformaldehyde for 15 minutes. Following a rinse in PBS, pH 7.4, endogenous peroxidase activity was quenched upon incubation for 30 minutes with $0.3 \% \mathrm{H}_{2} \mathrm{O}_{2}$ in absolute methanol, followed by a 6-minute rehydration in phosphate buffered saline (PBS). Slides were then incubated with $0.4 \%$ Triton-x 100 for 10 minutes. After incubation with blocking serum for 30 minutes at room temperature (4\% normal goat serum), sections were incubated with SSA6, a mouse monoclonal antibody against human $\beta 3$ integrin subunit (1:2000). The specificity of this antibody has been shown and confirmed by other authors [12,21]. Negative controls were analysed on adjacent sections incubated without primary antibody. A PBS rinse was followed by treatment with a secondary antibody consisting of biotinylated goat anti-mouse IgG antibody for 30 minutes (Vector Laboratories). After this incubation, sections were washed and incubated with avidin:biotinylated horseradish peroxidase macromolecular complex for 60 minutes. Diaminobenzidine (DAB) was added and incubated for 8 minutes to complete the reaction and to visualize the immunostaining. As a final step, sections were counterstained with hematoxylin for 5 minutes, dehydrated in a graded series of ethanols, and cleared with xylene. A coverslip was placed over Permount for evaluation by light microscopy. Negative external controls were obtained by withholding the primary antibody and positive internal controls were verified by the endometrial blood vessel staining. The resulting staining was evaluated using a Nikon microscope (Tokyo, Japan), by a single blinded observer (B.A.L.) without knowledge of the subject's group. Board certified pathologists of both institutions performed the endometrial dating according to Noyes's criteria [22]. Endometrial dating was considered as out-ofphase, when a discrepancy of 3 days or more was seen between endometrial dating and date of the menstrual cycle.

Assessment of staining intensity and distribution in endometrial glands was made using the semi-quantitative histologic score (HSCORE) system. HSCORE was calculated using the following equation: $\mathrm{HSCORE}=\Sigma \mathrm{Pi}(\mathrm{i}+1)$ / 100 , where $\mathrm{i}=$ intensity of staining with a value of 1,2 , or 3 , (weak, moderate or strong, respectively) and $\mathrm{Pi}=$ the percentage of stained endometrial epithelial cells for each intensity, varying from $0-100 \%$. Low intra-observer $(r=0.983 ; P<0.0001)$ and inter-observer $(r=0.994 ; P<$ $0.0001)$ differences for HSCORE in uterine tissues have been previously reported using this technique [23]. The numerical cut-off for a negative result for the $\alpha v \beta 3$ integrin was a HSCORE of $\leq 0.7$, based on previous ROC analysis [21]. Based on histological dating, in-phase or out-of-phase, and $\beta 3$ integrin expression, positive/negative, we proposed two types of defects: Type I defect is an out-of-phase endometrium with negative $\beta 3$ integrin subunit expression, and Type II defect is an "in-phase" endometrium with negative $\beta 3$ integrin subunit expression [11].

\section{Statistical analysis}

Sample size was calculated using the same parameters as previously published [24]; briefly, it was considered an $\alpha$ and $\beta$ error of 0.05 and 0.2 , respectively; variance of the expression of $\beta 3$ integrin was considered as 0.79 , and a minimal difference of HSCORE between groups was 1.4, i.e., the double of the 0.7 cut-off. These figures yielded, at least, 7 cases per group. Gaussian distribution was confirmed with D'Agostino \& Pearson omnibus normality test. If Gaussian distribution was confirmed, unpaired $t$-test was used; otherwise, it was used Mann-Whitney $U$ test. ANCOVA was used for adjusting HSCORE between groups, since age in controls and cases (recurrent pregnancy loss) was significantly different. ANCOVA online calculator (http://vassarstats.net/ancova2L.html) and GraphPad Prism 6.0 (GraphPad Software, San Diego, CA) were used for statistical analysis.

\section{Results}

Details of the population studied are depicted in Table 1. HSCORE in controls was significantly higher compared to the RPL group (Figure 1). The median (range) expression of $\beta 3$ in controls and cases was 1.94 (0 to 3.5) vs. 0.82 ( 0 to 3.6 ), respectively ( $P=0.001$; Mann Whitney $U$ test). ANCOVA analysis was conducted to identify if age between groups had influence on $\beta 3$ subunit integrin expression. Levene's test of equality of error variances was 0.7. After running ANCOVA analysis, age was adjusted, and the $P$ value between groups was 0.03 , confirming the significantly difference between groups, despite the age difference between groups.

\section{Morphological dating and comparison with integrin staining}

Compared to controls $(n=29)$, women with RPL ( $n=$ 21) showed more out-of-phase endometrium, i.e., histological delay in maturation. Only 10 out of 21 cases (47.6 - 95 CI: 28.3 to $67.6 \%$ ) were dated in-phase in RPL samples. In contrast, 26 out 29 controls were in-phase (89.7\% - 95 CI: 73.6 to 96.4\%). Likewise, positive expression of $\beta 3$ integrin subunit followed the same pattern in controls. Therefore positive expression of $\beta 3$ integrin subunit and an in-phase endometrium were present in 24 out of 29 controls ( $82.8 \%$ - 95 CI: 65.5 to $92.4 \%)$, while only 6 out of 21 RPL ( $28.6 \%-95$ CI: 13.8 to $50.0 \%$ ) samples could be considered "normal". While a type II defect, determined as in phase endometrium but negative 


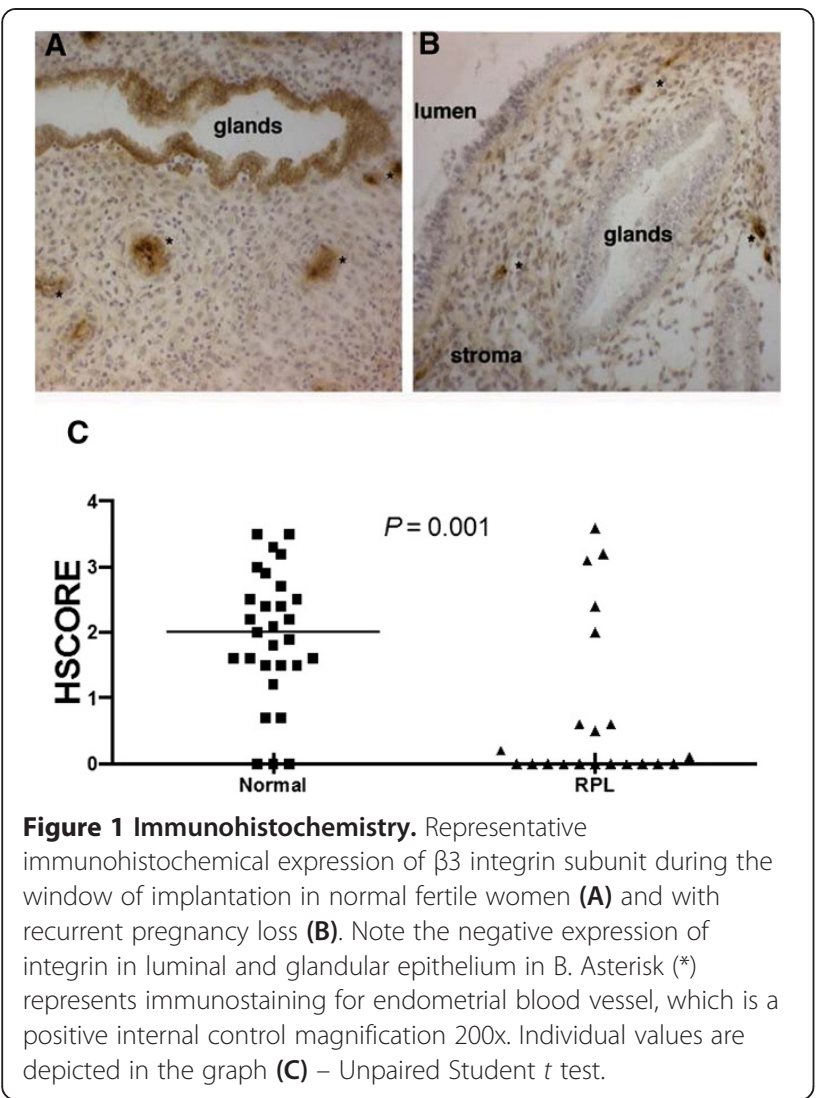

staining for $\beta 3$ integrin subunit, was found in $6.9 \%$ of control samples (CI: 1.9 to $22.0 \%$ ), $19.1 \%$ of RPL samples (CI: 7.7 to $40.0 \%$ ) were affected (Table 1 ). The most frequent defect seen in RPL samples $(P=0.0005)$ however was a type I defect with 11 of $21 \mathrm{RPL}$ samples $(52.4 \%$ - $95 \mathrm{CI}$ : 32.4 to $71.6 \%$ ) compared to only 3 out of 29 controls (10.3\% - 95CI: 3.6 to $26.4 \%$ ) (Table 1).

\section{Discussion}

The conflicting data on $\beta 3$ integrin subunit expression in endometrium of women with RPL led us to conduct the present study. Our data indicate that women with unexplained RPL have significantly reduced integrin expression compared to controls. Our results differ from those published from Xu et al. [15] and Tuckerman [17]. The probable cause for this discrepancy is the methodology. As it is known, integrin is a membrane adhesion protein and the use of FFPE technique yield artefacts [16], or the variability between observers [17]. We have shown that inexperienced observers in HSCORE tend to give higher HSCOREs [20].

Despite the majority of cases from the RPL group $(0.52$ - 95 CI: 0.32 to 0.71$)$ demonstrated an out-ofphase endometrium and a negative expression of integrin $\beta 3$ subunit (Type I defect), an additional 4 cases [0.19 (95 CI:0.07 to 0.4)] presented a type II defect, where integrin expression is negative in an "in-phase" endometrium. These findings demonstrate the need for further molecular analysis, besides the histological assessment of endometrial tissue. This finding is not new. Coutifaris et al. suggested that morphological criteria do not differ between fertile and infertile women [25]. Possible causes for this type II defect can be related to the presence of endometriosis [26]. As endometriosis is associated with reduced integrin expression [21], a highunknown incidence of endometriosis in women with recurrent abortions might be present, and the reduced expression of integrin could be the first clue. This hypothesis is supported by the findings that anti-lamin-1 antibody is reduced in women with endometriosis and with recurrent pregnancy loss [27]. In light of recent evidence linking endometriosis to progesterone resistance [28], these findings support a possible connection between occult or undiagnosed gynaecologic pathology and a loss of normal endometrial receptivity. The fact that integrin expression is reduced in RPL highlights the importance of this adhesion protein for implantation. This reduction is in accordance with other conditions related to infertility. For instance, low integrin expression was seen in women with different degrees of hydrosalpinges [24,29]. After removal of hydrosalpinges, levels of $\alpha v b 3$ integrin increase [30], as well as pregnancies rates [31].

The low expression of integrin in endometrium of women with RPL supports the role of this adhesion molecule in the feto-maternal communication during implantation. In patients with RPL, this reduction of integrin expression may be due to the fact that HOXA10, another implantation marker, regulates the expression of $\beta 3$ integrin [32]. HOXA10 has been shown to be a modulator of integrin $\beta 3$, and could be deregulated in endometrium of women with RPL, as suggested by animal studies [33].

There are a few weaknesses in this study. Laparoscopy was not routinely performed in these patients to investigate peritoneal causes such as endometriosis. Likewise, karyotyping was not performed, mainly because it has a low yield for abnormalities (1 to $2 \%$ ).

The appropriate statistical care, sample size and the appropriate immunohistochemical analysis with a wellestablished antibody are strengths of this study and corroborate to the accuracy of the results.

\section{Conclusions}

In conclusion, we demonstrate that women with RPL present an endometrial defect during the window of implantation, more specifically, a reduced expression of $\beta 3$ integrin subunit. Such defects comprised both "in-phase" and "out-of-phase" histology. Further prospective studies are needed to better determine the cause and treatment of these types of endometrial receptivity defects in women with otherwise unexplained RPL. If this correlation 
remains to be seen, women with unexplained RPL may benefit from additional laparoscopic examination in order to discover potential gynaecological conditions.

\section{Competing interests}

The authors declare that they have no competing interests.

\section{Authors' contributions}

AG participated in the study design, drafted the manuscript \& participated in tissue collection. RFS helped draft the manuscript and performed the statistical analysis. JJ participated in tissue collection and processing. BL conceived of the study, participated in its design and coordination, participated in tissue collection, reviewed the manuscript \& performed the Immunohistochemistry staining and analysis. All authors read and approved the final manuscript.

\section{Acknowledgements}

We would like to acknowledge the assistance with data entry by Lauren Nelson and Ashley Graham. Furthermore, we acknowledge financial support by Deutsche Forschungsgemeinschaft and Ruprecht-Karls-Universität Heidelberg within the funding programme Open Access Publishing.

\section{Author details}

${ }^{1}$ Department of Gynecological Endocrinology and Reproductive Medicine, University Hospital Heidelberg, Heidelberg, Germany. ${ }^{2}$ Departamento de Ginecologia e Obstetrícia, Universidade Federal do Rio Grande do Sul, Porto Alegre, Brazil. ${ }^{3}$ Greenville University Medical Center, Greenville, SC, USA.

Received: 10 March 2014 Accepted: 10 June 2014

Published: 20 June 2014

\section{References}

1. Ford HB, Schust DJ: Recurrent pregnancy loss: etiology, diagnosis, and therapy. Rev Obstet Gynecol 2009, 2:76-83.

2. Kwak-Kim J, Bao S, Lee SK, Kim JW, Gilman-Sachs A: Immunological modes of pregnancy loss: inflammation, immune effectors, and stress. Am J Reprod Immunol 2014, doi:10.1111/aji.12234. [Epub ahead of print].

3. Larsen EC, Christiansen OB, Kolte AM, Macklon N: New insights into mechanisms behind miscarriage. BMC Med 2013, 11:154.

4. Wilcox AJ, Baird DD, Weinberg CR: Time of implantation of the conceptus and loss of pregnancy. N Engl J Med 1999, 340:1796-1799.

5. Navot D, Scott RT, Droesch K, Veeck LL, Liu HC, Rosenwaks Z: The window of embryo transfer and the efficiency of human conception in vitro. Fertil Steril 1991, 55:114-118.

6. Serle E, Aplin JD, Li TC, Warren MA, Graham RA, Seif MW, Cooke ID: Endometrial differentiation in the peri-implantation phase of women with recurrent miscarriage: a morphological and immunohistochemical study. Fertil Steril 1994, 62:989-996.

7. Othman R, Omar MH, Shan LP, Shafiee MN, Jamal R, Mokhtar NM: Microarray profiling of secretory-phase endometrium from patients with recurrent miscarriage. Reprod Biol 2012, 12:183-199.

8. Kao LC, Tulac S, Lobo S, Imani B, Yang JP, Germeyer A, Osteen K, Taylor RN Lessey BA, Giudice LC: Global gene profiling in human endometrium during the window of implantation. Endocrinology 2002, 143:2119-2138.

9. Najwa AR, Sengupta J, Ghosh D: A systems biology approach towards understanding the process of blastocyst implantation. Indian J Physiol Pharmacol 2009, 53:197-208.

10. Talbi S, Hamilton AE, Vo KC, Tulac S, Overgaard MT, Dosiou C, Le Shay N, Nezhat CN, Kempson R, Lessey BA, Nayak NR, Giudice LC: Molecular phenotyping of human endometrium distinguishes menstrual cycle phases and underlying biological processes in normo-ovulatory women. Endocrinology 2006, 147:1097-1121.

11. Lessey BA, Castelbaum AJ, Sawin SW, Sun J: Integrins as markers of uterine receptivity in women with primary unexplained infertility. Fertil Steril 1995, 63:535-542.

12. Lessey BA, Damjanovich L, Coutifaris C, Castelbaum A, Albelda SM, Buck CA: Integrin adhesion molecules in the human endometrium. Correlation with the normal and abnormal menstrual cycle. J Clin Invest 1992, 90:188-195.

13. Lessey BA, Castelbaum AJ, Buck CA, Lei Y, Yowell CW, Sun J: Further characterization of endometrial integrins during the menstrual cycle and in pregnancy. Fertil Steril 1994, 62:497-506.
14. Lessey BA: Implantation defects in infertile women with endometriosis. Ann N Y Acad Sci 2002, 955:265-280. discussion 293-5, 396-406.

15. $X u B$, Sun $X$, Li L, Wu L, Zhang A, Feng Y: Pinopodes, leukemia inhibitory factor, integrin-beta3, and mucin-1 expression in the peri-implantation endometrium of women with unexplained recurrent pregnancy loss. Fertil Steril 2012, 98:389-395.

16. Lessey BA, Castelbaum AJ: Integrins and endometriosis: fact or artefact? Hum Reprod 1998, 13:3578-3580.

17. Tuckerman EM, Laird SM, Prakash A, Li TC: Expression of integrins in the endometrium of women with recurrent miscarriage. Fertil Steril 2006, 86:755-757.

18. Coughlan C, Sinagra M, Ledger W, Li TC, Laird S: Endometrial integrin expression in women with recurrent implantation failure after in vitro fertilization and its relationship to pregnancy outcome. Fertil Steril 2013, 100:825-830.

19. Quenby S, Anim-Somuah M, Kalumbi C, Farquharson R, Aplin JD: Different types of recurrent miscarriage are associated with varying patterns of adhesion molecule expression in endometrium. Reprod Biomed Online 2007, 14:224-234.

20. Fuhrich DG, Lessey BA, Savaris RE: Comparison of HSCORE assessment of endometrial beta3 integrin subunit expression with digital HSCORE using computerized image analysis (ImageJ). Anal Quant Cytol Histol 2013, 35:210-216

21. Lessey BA, Castelbaum AJ, Sawin SW, Buck CA, Schinnar R, Bilker W, Strom $B L:$ Aberrant integrin expression in the endometrium of women with endometriosis. J Clin Endocrinol Metab 1994, 79:643-649.

22. Noyes RW, Hertig AT, Rock J: Dating the endometrial biopsy. Fertil Steril 1950, 1:3-25.

23. Budwit-Novotny DA, McCarty KS, Cox EB, Soper JT, Mutch DG, Creasman WT, Flowers JL, McCarty KSJ: Immunohistochemical analyses of estrogen receptor in endometrial adenocarcinoma using a monoclonal antibody. Cancer Res 1986, 46:5419-5425.

24. Savaris RF, Pedrini JL, Flores R, Fabris G, Zettler CG: Expression of alpha 1 and beta 3 integrins subunits in the endometrium of patients with tubal phimosis or hydrosalpinx. Fertil Steril 2006, 85:188-192.

25. Coutifaris C, Myers ER, Guzick DS, Diamond MP, Carson SA, Legro RS, McGovern PG, Schlaff WD, Carr BR, Steinkampf MP, Silva S, Vogel DL, Leppert PC: Histological dating of timed endometrial biopsy tissue is not related to fertility status. Fertil Steril 2004, 82:1264-1272.

26. Lessey BA, Young SL: Integrins and other cell adhesion molecules in endometrium and endometriosis. Semin Reprod Endocrinol 1997, 15:291-299.

27. Inagaki J, Kondo A, Lopez LR, Shoenfeld Y, Matsuura E: Pregnancy loss and endometriosis: pathogenic role of anti-laminin-1 autoantibodies. Ann N Y Acad Sci 2005, 1051:174-184.

28. Aghajanova L, Velarde MC, Giudice LC: Altered gene expression profiling in endometrium: evidence for progesterone resistance. Semin Reprod Med 2010, 28:51-58.

29. Meyer WR, Castelbaum AJ, Somkuti S, Sagoskin AW, Doyle M, Harris JE, Lessey BA: Hydrosalpinges adversely affect markers of endometrial receptivity. Hum Reprod 1997, 12:1393-1398.

30. Bildirici I, Bukulmez O, Ensari A, Yarali H, Gurgan T: A prospective evaluation of the effect of salpingectomy on endometrial receptivity in cases of women with communicating hydrosalpinges. Hum Reprod 2001 $16: 2422-2426$

31. Sagoskin AW, Lessey BA, Mottla GL, Richter KS, Chetkowski RJ, Chang AS, Levy MJ, Stillman RJ: Salpingectomy or proximal tubal occlusion of unilateral hydrosalpinx increases the potential for spontaneous pregnancy. Hum Reprod 2003, 18:2634-2637.

32. Daftary GS, Troy PJ, Bagot CN, Young SL, Taylor HS: Direct regulation of beta3-integrin subunit gene expression by HOXA10 in endometrial cells. Mol Endocrinol 2002, 16:571-579.

33. Yao MW, Lim H, Schust DJ, Choe SE, Farago A, Ding Y, Michaud S, Church $G M$, Maas RL: Gene expression profiling reveals progesterone-mediated cell cycle and immunoregulatory roles of Hoxa-10 in the preimplantation uterus. Mol Endocrinol 2003, 17:610-627.

doi:10.1186/1477-7827-12-53

Cite this article as: Germeyer et al.: Endometrial beta3 Integrin profile reflects endometrial receptivity defects in women with unexplained recurrent pregnancy loss. Reproductive Biology and Endocrinology 2014 12:53. 